\title{
Compound synchronization of fourth-order memristor oscillator
}

Ailong $\mathrm{Wu}^{1,2,3}$ and Jine Zhang ${ }^{1 *}$

"Correspondence: hbnufu@yeah.net

${ }^{1}$ College of Mathematics and Statistics, Hubei Normal University, Huangshi, 435002, China Full list of author information is available at the end of the article

\begin{abstract}
The conception of memristor lead to a new approach in nonlinear circuit design. In this paper, the compound synchronization of the fourth-order memristor oscillator is studied. The proposed scheme of compound synchronization is described by three drive systems (a scaling drive system, two base drive systems) and one response system. The version of synchronization is advantageous in circuit application due to the novel structure. As a generalization of the obtained results, secure communication via compound synchronization is discussed in detail.
\end{abstract}

Keywords: memristor; hybrid systems; circuit analysis; compound synchronization

\section{Introduction}

Recently, lots of nonlinear memristor oscillators based on Chua's circuits have been proposed [1-10]. The feature of pinched hysteresis in memristor oscillators has directed a lot of attention to this exciting field. In these circuit implementations, the conventional Chua diode is replaced by nonlinear memristors. The analytical and emulational results demonstrate unprecedented phenomena in the field of memristor oscillators, which may represent a new paradigm in the theory, design and application of electronic circuits [1$4,6,8,10]$. The unprecedented phenomena have successfully been applied into chaotic circuits, sensitive control systems, etc. $[4,6,8]$. Many researchers are exploring a variety of technologies to accelerate the development of memristor oscillators. The nonlinear characteristic of memristor inhibits the complexity of the analysis [11-13]. A number of scholars are rethinking new theories, techniques and methods to analyze and investigate such two-terminal passive element. With the flourishing applications, they had to uncork new bottlenecks.

Based on the works in [4] and [6], in this paper, consider a fourth-order memristor oscillator with its dynamics described by the following equations:

$$
\left\{\begin{array}{l}
\dot{\varphi}(t)=v_{1}(t), \\
\dot{v}_{1}(t)=\frac{1}{C_{1} R} v_{2}(t)-\frac{1}{C_{1} R} v_{1}(t)-\frac{1}{C_{1}} W(\varphi(t)) v_{1}(t), \\
\dot{v}_{2}(t)=\frac{1}{C_{2} R} v_{1}(t)-\frac{1}{C_{2} R} v_{2}(t)-\frac{1}{C_{2}} \ell(t), \\
\dot{\ell}(t)=\frac{1}{L} v_{2}(t),
\end{array}\right.
$$

where $v_{1}(t)$ and $v_{2}(t)$ denote voltages, $C_{1}$ and $C_{2}$ represent capacitors, $W(\varphi(t))$ is memductance function, $\varphi(t), \ell(t), R$, and $L$ are magnetic flux, current, resistor, and inductor, respectively.

(2014 Wu and Zhang; licensee Springer. This is an Open Access article distributed under the terms of the Creative Commons Attribution License (http://creativecommons.org/licenses/by/2.0), which permits unrestricted use, distribution, and reproduction in any medium, provided the original work is properly cited. 
Using the mathematical model of the cubic memristor $[1,2,8]$, the memductance function is given by

$$
W(\varphi(t))=a+3 b \varphi(t)^{2}
$$

where $a$ and $b$ are parameters. Similarly as in Reference [4], $a$ and $b$ denote slope. According to the characteristic of the two-terminal memristor circuit (see, e.g., Itoh and Chua [4], p.3184, p.3185), the values of $a$ and $b$ could be positive or negative.

The synchronization of memristor oscillator plays an important role in chaos control and its application $[8,11]$. We notice that synchronization of complex systems and complex networks has received significant attention [14-49]. In particular, complete synchronization [11, 14-18], anti-synchronization [19-24], phase synchronization [16, 25-27], lag synchronization [17, 28-36], projective synchronization [17, 37-45], and combination synchronization $[46,47]$ have attracted phenomenal worldwide attention in view of many potential applications. On applying the memristor oscillator to secure communication, the typical approach is to transmit the information signal by means of one chaotic system. This way is a simple approach that can be used in some cases, but definitely not in all. An optimal design of secure communication via the memristor oscillator needs to improve the complexity level of the driving signal and the modulation scheme used. Can we compound multiple memristor oscillators to transmit the information signal? With such a target in mind, it would be extremely helpful to develop some effective methods capturing the behaviors of distinct memristor oscillators, in order to strengthen the security of communication. Meanwhile, theoretical investigation can help to interpret the experimental observations and predict complex circuit behavior.

Motivated by the above discussions, based on some ideas borrowed from the compound design approach [8], our aim in this paper is to explore the compound synchronization of the fourth-order memristor oscillator (1). Some new and effective synchronization criteria are proposed for fourth-order memristor oscillator (1). The derived results show that the compound synchronization is an efficient solver for chaos control and its application. In fact, the compound synchronization is capable to capture a wide class of memristor dynamics. In the scheme of compound synchronization, the drive systems are divided into two categories: scaling drive system and base drive system, which is entirely different from the conventional synchronization scheme. The scheme of compound synchronization is then successfully extended to the study of secure communication.

The remaining part of this paper consists of four sections. Section 2 describes some preliminaries. The main theoretical results are stated in Section 3. Secure communication via compound synchronization is given in Section 4. Finally, concluding remarks are made in Section 5.

\section{Preliminaries}

It is useful to first introduce the scheme of compound synchronization that is needed later.

Generally, compound synchronization is based on a scaling drive system, multiple base drive systems and one response system. In some engineering applications and hardware implementations, compound synchronization constituting of a scaling drive system, two base drive systems and one response system is usually considered. Figure 1 describes a schematic of compound synchronization scheme. In fact, compound synchronization has 


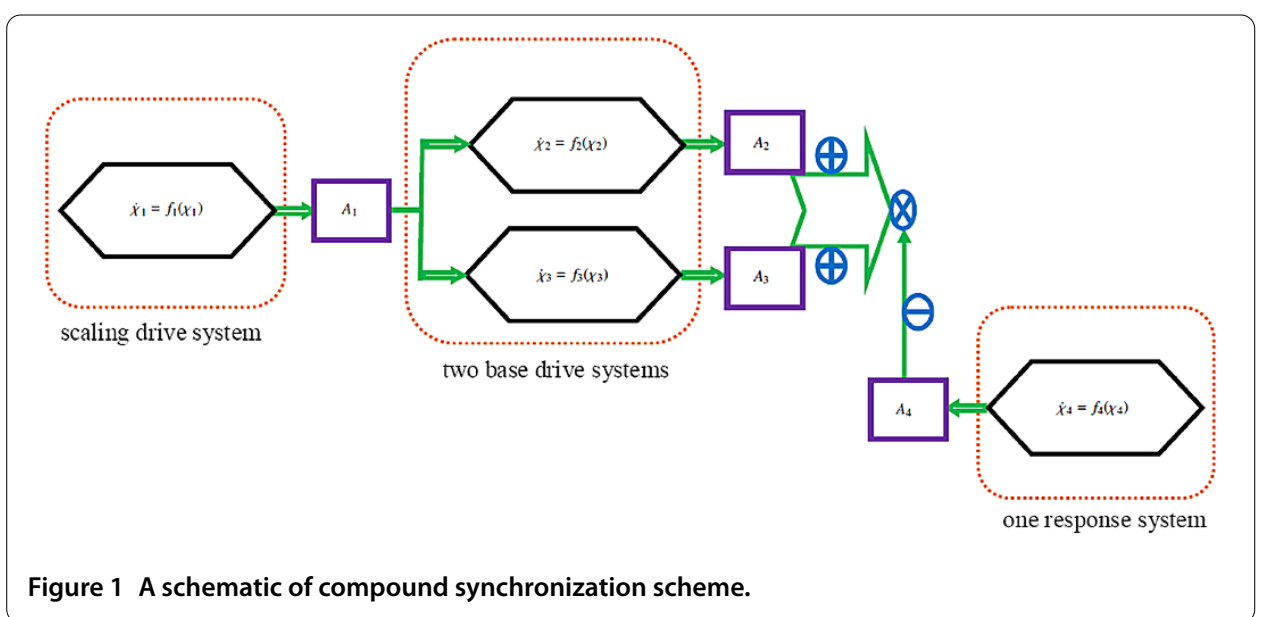

its particular physical meaning. For example, in Figure 1, the scaling drive system scales the synthetic signals of two base drive systems, generating resultant signals, then the response system tracks the resultant signals.

Next, we give specific mathematical descriptions of compound synchronization scheme.

Consider the scaling drive system

$$
\dot{\chi}_{1}=f_{1}\left(\chi_{1}\right)
$$

The two base drive systems are given by

$$
\begin{aligned}
& \dot{\chi}_{2}=f_{2}\left(\chi_{2}\right), \\
& \dot{\chi}_{3}=f_{3}\left(\chi_{3}\right),
\end{aligned}
$$

and one response system is described by

$$
\dot{\chi}_{4}=f_{4}\left(\chi_{4}\right)+u,
$$

where we have the state vectors $\chi_{1}=\left(\chi_{11}, \chi_{12}, \ldots, \chi_{1 n}\right)^{T}, \chi_{2}=\left(\chi_{21}, \chi_{22}, \ldots, \chi_{2 n}\right)^{T}, \chi_{3}=$ $\left(\chi_{31}, \chi_{32}, \ldots, \chi_{3 n}\right)^{T}, \chi_{4}=\left(\chi_{41}, \chi_{42}, \ldots, \chi_{4 n}\right)^{T}$, the vector functions $f_{1}(\cdot), f_{2}(\cdot), f_{3}(\cdot), f_{4}(\cdot): \Re^{n} \rightarrow$ $\Re^{n}$, and $u=\left(u_{1}, u_{2}, \ldots, u_{n}\right)^{T}: \Re^{n} \times \Re^{n} \times \cdots \times \Re^{n} \rightarrow \Re^{n}$ is the appropriate control input that will be designed in order to obtain a certain control objective.

Definition 1 The drive systems (3)-(5) are said to compound synchronize with the response system (6) if there exist $n$-dimensional constant diagonal matrices $A_{1}, A_{2}, A_{3}$, and $A_{4} \neq 0$ such that

$$
\lim _{t \rightarrow+\infty}\|e\|=\lim _{t \rightarrow+\infty}\left\|A_{1} X_{1}\left(A_{2} X_{2}+A_{3} X_{3}\right)-A_{4} X_{4}\right\|=0
$$

where $\|\cdot\|$ is the vector norm, and $e=\left(e_{1}, e_{2}, \ldots, e_{n}\right)^{T}$ is the synchronization error vector; we have $X_{1}=\operatorname{diag}\left(\chi_{11}, \chi_{12}, \ldots, \chi_{1 n}\right), X_{2}=\operatorname{diag}\left(\chi_{21}, \chi_{22}, \ldots, \chi_{2 n}\right), X_{3}=\operatorname{diag}\left(\chi_{31}, \chi_{32}, \ldots, \chi_{3 n}\right)$, $X_{4}=\operatorname{diag}\left(\chi_{41}, \chi_{42}, \ldots, \chi_{4 n}\right)$. 
Remark 1 As stated earlier, according to Definition 1, the physical implication of compound synchronization is rather intuitive. The synthetic signals of two base drive systems (4) and (5) are scaled via the scaling drive system (3), then the response system (6) tracks the resultant signals of drive systems (3)-(5).

Remark 2 In Definition 1, matrices $A_{1}, A_{2}, A_{3}$, and $A_{4}$ are often called the scaling matrices. It is not hard to find that compound synchronization contains the multiplication of the scaling drive system and multiple base drive systems. Moreover, the drive systems in the scheme of compound synchronization can be completely identical or different.

Remark 3 The scheme of the compound synchronization is an improvement and extension of the existing synchronization schemes in the literature. When the scaling matrices $A_{1} \neq 0, A_{2}=0$ or $A_{3}=0$, the compound synchronization will degrade into a kind of function projective synchronization. When the scaling matrices $A_{1}=0$ or $A_{2}=A_{3}=0$, the compound synchronization will change into chaos control. In addition, if the scaling drive system (3) is removed, then the compound synchronization will be reduced to combination synchronization. If the base drive systems (4) and (5) are removed, then the compound synchronization will be reduced to complete synchronization.

\section{Theoretical results}

In this section, we are in the position to investigate our main theoretical results.

From (1) and (2), it follows that

$$
\left\{\begin{array}{l}
\dot{\varphi}(t)=v_{1}(t) \\
\dot{v}_{1}(t)=\frac{1}{C_{1} R} v_{2}(t)-\frac{1}{C_{1} R} v_{1}(t)-\frac{a}{C_{1}} v_{1}(t)-\frac{3 b}{C_{1}} \varphi(t)^{2} v_{1}(t), \\
\dot{v}_{2}(t)=\frac{1}{C_{2} R} v_{1}(t)-\frac{1}{C_{2} R} v_{2}(t)-\frac{1}{C_{2}} \ell(t) \\
\dot{\ell}(t)=\frac{1}{L} \nu_{2}(t)
\end{array}\right.
$$

By merging similar items,

$$
\left\{\begin{array}{l}
\dot{\varphi}(t)=v_{1}(t) \\
\dot{v}_{1}(t)=\frac{1}{C_{1} R} v_{2}(t)-\left[\frac{1}{C_{1} R}+\frac{a}{C_{1}}\right] v_{1}(t)-\frac{3 b}{C_{1}} \varphi(t)^{2} v_{1}(t), \\
\dot{v}_{2}(t)=\frac{1}{C_{2} R} v_{1}(t)-\frac{1}{C_{2} R} v_{2}(t)-\frac{1}{C_{2}} \ell(t) \\
\dot{\ell}(t)=\frac{1}{L} v_{2}(t)
\end{array}\right.
$$

In order to facilitate discussion, we would need to make a rewrite for (9). Let $x_{11}(t)=\varphi(t)$, $x_{12}(t)=v_{1}(t), x_{13}(t)=v_{2}(t), x_{14}(t)=\ell(t), \alpha_{1}=\frac{1}{C_{1} R}, \alpha_{2}=\frac{1}{C_{1} R}+\frac{a}{C_{1}}, \alpha_{3}=\frac{3 b}{C_{1}}, \alpha_{4}=\frac{1}{C_{2} R}, \alpha_{5}=\frac{1}{C_{2}}$, $\alpha_{6}=\frac{1}{L}$, then (9) is equivalent to

$$
\left\{\begin{array}{l}
\dot{x}_{11}=x_{12} \\
\dot{x}_{12}=\alpha_{1} x_{13}-\alpha_{2} x_{12}-\alpha_{3} x_{11}^{2} x_{12} \\
\dot{x}_{13}=\alpha_{4} x_{12}-\alpha_{4} x_{13}-\alpha_{5} x_{14} \\
\dot{x}_{14}=\alpha_{6} x_{13}
\end{array}\right.
$$

Remark 4 System (9) is a vectorization system. To facilitate the discussion, by abandoning dimension, in the subsequent discussion, we will study the rewritten system (10). Meanwhile, since $C_{1}$ and $C_{2}$ represent capacitors, $R$ denotes resistor, $L$ denotes inductor, the 


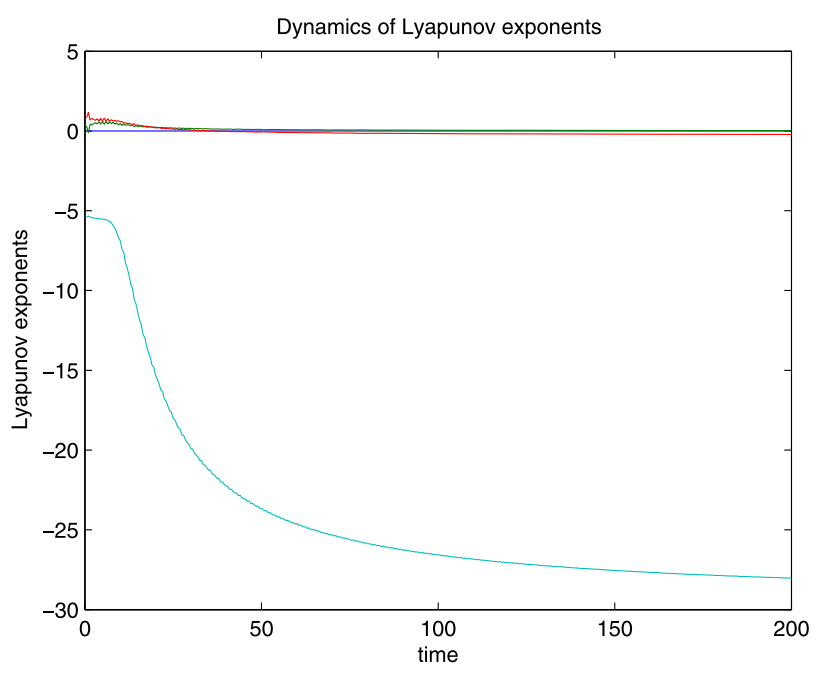

Figure 2 Dynamics of Lyapunov exponents from the fourth-order memristor oscillator.

values of $\alpha_{1}, \alpha_{4}, \alpha_{5}$, and $\alpha_{6}$ must be positive numbers. Generally, as the most characteristic feature of the two-terminal memristor circuit (see, e.g., Itoh and Chua [4], p.3184), the slopes $a$ and $b$ are positive numbers, then the values of $\alpha_{2}$ and $\alpha_{3}$ are also positive.

Choosing the parameters $\alpha_{1}=16.4, \alpha_{2}=3.28, \alpha_{3}=19.7, \alpha_{4}=1, \alpha_{5}=1, \alpha_{6}=15$, the initial state $x_{11}(0)=0, x_{12}(0)=0, x_{13}(0)=0, x_{14}(0)=0.02$, by means of a computer program of MATLAB, the corresponding Lyapunov exponents of system (10) are 0.019005 , $0,-0.213441,-28.032608$. The numerical result is described in Figure 2. Clearly, there is one positive Lyapunov exponent, which implies that system (10) is chaotic. In fact, system (10) indeed generates chaotic behavior. Using MATLAB, we get the results shown in Figure 3.

Remark 5 Sun et al. [8] have discussed the compound design of memristor chaotic oscillator system and obtained some interesting results. In [8], the memristor oscillator model is based on the circuit model in [2]. Thus, the scaling drive system is as follows:

$$
\left\{\begin{array}{l}
\dot{y}_{11}=y_{12}, \\
\dot{y}_{12}=\varrho_{1} y_{13}+\varrho_{2} y_{12}-\varrho_{3} y_{11}^{2} y_{12}, \\
\dot{y}_{13}=y_{12}-y_{13}+y_{14}, \\
\dot{y}_{14}=-\varrho_{4} y_{13}-\varrho_{5} y_{14} .
\end{array}\right.
$$

By comparing the scaling drive system in [8] with the scaling drive system (10) in this paper, it is easy to find that the scale factors on the third equation of the scaling drive system in [8] are not imported. Conversely, in this paper, the introduced memristor oscillator system is based on the circuit model in [6], the scale factors are fully considered. What is more, the circuit of (1) has more superior structure performances than the memristor chaotic oscillator system in [8]. And then the scaling drive system (10) in this paper has more a comprehensive and practical meaning. In fact, these scale factors play a significant role in the design and implementation of the control scheme. 


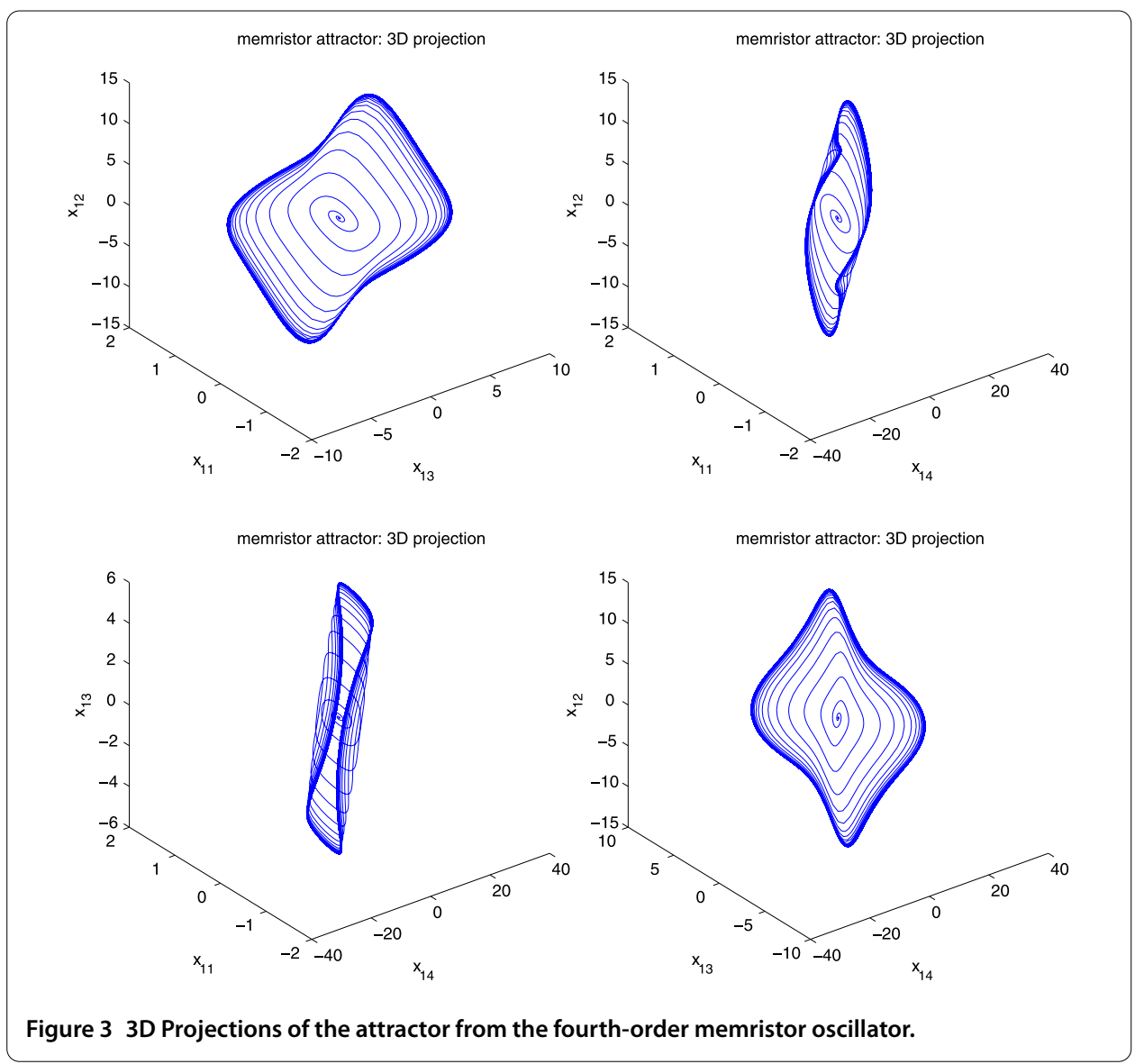

Considering (10) as the scaling drive system, according to compound synchronization scheme, obviously, we can choose the first base drive system described by

$$
\left\{\begin{array}{l}
\dot{x}_{21}=x_{22} \\
\dot{x}_{22}=\beta_{1} x_{23}-\beta_{2} x_{22}-\beta_{3} x_{21}^{2} x_{22}, \\
\dot{x}_{23}=\beta_{4} x_{22}-\beta_{4} x_{23}-\beta_{5} x_{24}, \\
\dot{x}_{24}=\beta_{6} x_{23}
\end{array}\right.
$$

the second base drive system is given by

$$
\left\{\begin{array}{l}
\dot{x}_{31}=x_{32} \\
\dot{x}_{32}=\gamma_{1} x_{33}-\gamma_{2} x_{32}-\gamma_{3} x_{31}^{2} x_{32} \\
\dot{x}_{33}=\gamma_{4} x_{32}-\gamma_{4} x_{33}-\gamma_{5} x_{34} \\
\dot{x}_{34}=\gamma_{6} x_{33}
\end{array}\right.
$$

and the response system is described by

$$
\left\{\begin{array}{l}
\dot{x}_{41}=x_{42}+u_{1}, \\
\dot{x}_{42}=\eta_{1} x_{43}-\eta_{2} x_{42}-\eta_{3} x_{41}^{2} x_{42}+u_{2}, \\
\dot{x}_{43}=\eta_{4} x_{42}-\eta_{4} x_{43}-\eta_{5} x_{44}+u_{3}, \\
\dot{x}_{44}=\eta_{6} x_{43}+u_{4},
\end{array}\right.
$$


where $\beta_{1}, \beta_{2}, \beta_{3}, \beta_{4}, \beta_{5}, \beta_{6}, \gamma_{1}, \gamma_{2}, \gamma_{3}, \gamma_{4}, \gamma_{5}, \gamma_{6}, \eta_{1}, \eta_{2}, \eta_{3}, \eta_{4}, \eta_{5}$, and $\eta_{6}$ are parameters, and $u_{1}, u_{2}, u_{3}$, and $u_{4}$ are the control inputs to be designed.

In our synchronization scheme, denote $A_{1}=\operatorname{diag}\left(a_{11}, a_{12}, a_{13}, a_{14}\right), A_{2}=\operatorname{diag}\left(a_{21}, a_{22}\right.$, $\left.a_{23}, a_{24}\right), A_{3}=\operatorname{diag}\left(a_{31}, a_{32}, a_{33}, a_{34}\right)$, and $A_{4}=\operatorname{diag}\left(a_{41}, a_{42}, a_{43}, a_{44}\right)$, thus

$$
\left\{\begin{array}{l}
e_{1}=a_{11} x_{11}\left(a_{21} x_{21}+a_{31} x_{31}\right)-a_{41} x_{41}, \\
e_{2}=a_{12} x_{12}\left(a_{22} x_{22}+a_{32} x_{32}\right)-a_{42} x_{42}, \\
e_{3}=a_{13} x_{13}\left(a_{23} x_{23}+a_{33} x_{33}\right)-a_{43} x_{43} \\
e_{4}=a_{14} x_{14}\left(a_{24} x_{24}+a_{34} x_{34}\right)-a_{44} x_{44}
\end{array}\right.
$$

Obviously, we have

$$
\left\{\begin{array}{l}
\dot{e}_{1}=a_{11} \dot{x}_{11}\left(a_{21} x_{21}+a_{31} x_{31}\right)+a_{11} x_{11}\left(a_{21} \dot{x}_{21}+a_{31} \dot{x}_{31}\right)-a_{41} \dot{x}_{41}, \\
\dot{e}_{2}=a_{12} \dot{x}_{12}\left(a_{22} x_{22}+a_{32} x_{32}\right)+a_{12} x_{12}\left(a_{22} \dot{x}_{22}+a_{32} \dot{x}_{32}\right)-a_{42} \dot{x}_{42}, \\
\dot{e}_{3}=a_{13} \dot{x}_{13}\left(a_{23} x_{23}+a_{33} x_{33}\right)+a_{13} x_{13}\left(a_{23} \dot{x}_{23}+a_{33} \dot{x}_{33}\right)-a_{43} \dot{x}_{43}, \\
\dot{e}_{4}=a_{14} \dot{x}_{14}\left(a_{24} x_{24}+a_{34} x_{34}\right)+a_{14} x_{14}\left(a_{24} \dot{x}_{24}+a_{34} \dot{x}_{34}\right)-a_{44} \dot{x}_{44} .
\end{array}\right.
$$

Combining with (10)-(13), the synchronization error system (15) can be transformed into the following form:

$$
\left\{\begin{aligned}
\dot{e}_{1}= & a_{11} x_{12}\left(a_{21} x_{21}+a_{31} x_{31}\right)+a_{11} x_{11}\left(a_{21} x_{22}+a_{31} x_{32}\right)-a_{41}\left(x_{42}+u_{1}\right), \\
\dot{e}_{2}= & a_{12}\left(\alpha_{1} x_{13}-\alpha_{2} x_{12}-\alpha_{3} x_{11}^{2} x_{12}\right)\left(a_{22} x_{22}+a_{32} x_{32}\right) \\
& +a_{12} x_{12}\left[a_{22}\left(\beta_{1} x_{23}-\beta_{2} x_{22}-\beta_{3} x_{21}^{2} x_{22}\right)+a_{32}\left(\gamma_{1} x_{33}-\gamma_{2} x_{32}-\gamma_{3} x_{31}^{2} x_{32}\right)\right] \\
& -a_{42}\left(\eta_{1} x_{43}-\eta_{2} x_{42}-\eta_{3} x_{41}^{2} x_{42}+u_{2}\right), \\
\dot{e}_{3}= & a_{13}\left(\alpha_{4} x_{12}-\alpha_{4} x_{13}-\alpha_{5} x_{14}\right)\left(a_{23} x_{23}+a_{33} x_{33}\right) \\
& +a_{13} x_{13}\left[a_{23}\left(\beta_{4} x_{22}-\beta_{4} x_{23}-\beta_{5} x_{24}\right)+a_{33}\left(\gamma_{4} x_{32}-\gamma_{4} x_{33}-\gamma_{5} x_{34}\right)\right] \\
& -a_{43}\left(\eta_{4} x_{42}-\eta_{4} x_{43}-\eta_{5} x_{44}+u_{3}\right), \\
\dot{e}_{4}= & a_{14} \alpha_{6} x_{13}\left(a_{24} x_{24}+a_{34} x_{34}\right)+a_{14} x_{14}\left(a_{24} \beta_{6} x_{23}+a_{34} \gamma_{6} x_{33}\right) \\
& -a_{44}\left(\eta_{6} x_{43}+u_{4}\right) .
\end{aligned}\right.
$$

Theorem 1 The drive systems (10)-(12) compound synchronize with the response system (13) if the control input is designed as

$$
\left\{\begin{aligned}
u_{1}= & \frac{1}{a_{41}}\left[a_{11} x_{11}\left(a_{21} x_{22}+a_{31} x_{32}+a_{21} x_{21}+a_{31} x_{31}\right)+a_{11} x_{12}\left(a_{21} x_{21}+a_{31} x_{31}\right)\right. \\
& +\alpha_{1} a_{12} x_{12}\left(a_{22} x_{22}+a_{32} x_{32}\right)-\eta_{1} a_{14} x_{14}\left(a_{24} x_{24}+a_{34} x_{34}\right) \\
& \left.-\left(a_{41}+\alpha_{1} a_{42}\right) x_{42}+\eta_{1} a_{44} x_{44}\right], \\
u_{2}= & \frac{1}{a_{42}}\left(\Theta-\alpha_{1}\left[a_{11} x_{11}\left(a_{21} x_{21}+a_{31} x_{31}\right)-a_{41} x_{41}\right]\right. \\
& +\left[a_{12} x_{12}\left(a_{22} x_{22}+a_{32} x_{32}\right)-a_{42} x_{42}\right] \\
& \left.+\alpha_{2}\left[a_{13} x_{13}\left(a_{23} x_{23}+a_{33} x_{33}\right)-a_{43} x_{43}\right]\right), \\
u_{3}= & \frac{1}{a_{43}}\left(\widetilde{\Theta}-\alpha_{2}\left[a_{12} x_{12}\left(a_{22} x_{22}+a_{32} x_{32}\right)-a_{42} x_{42}\right]\right. \\
& +\left[a_{13} x_{13}\left(a_{23} x_{23}+a_{33} x_{33}\right)-a_{43} x_{43}\right] \\
& \left.+\alpha_{3}\left[a_{14} x_{14}\left(a_{24} x_{24}+a_{34} x_{34}\right)-a_{44} x_{44}\right]\right), \\
u_{4}= & \frac{1}{a_{44}}\left[\eta_{1} a_{11} x_{11}\left(a_{21} x_{21}+a_{31} x_{31}\right)-\alpha_{3} a_{13} x_{13}\left(a_{23} x_{23}+a_{33} x_{33}\right)\right. \\
& +\alpha_{6} a_{14} x_{13}\left(a_{24} x_{24}+a_{34} x_{34}\right) \\
& +a_{14} x_{14}\left(\beta_{6} a_{24} x_{23}+a_{24} x_{24}\right)+a_{14} x_{14}\left(\gamma_{6} a_{34} x_{33}+a_{34} x_{34}\right) \\
& \left.-\eta_{1} a_{41} x_{41}-\alpha_{4} a_{43} x_{43}-\eta_{6} a_{44} x_{43}-a_{44} x_{44}\right],
\end{aligned}\right.
$$


where

$$
\begin{aligned}
\Theta= & a_{12}\left(\alpha_{1} x_{13}-\alpha_{2} x_{12}-\alpha_{3} x_{11}^{2} x_{12}\right)\left(a_{22} x_{22}+a_{32} x_{32}\right) \\
& +a_{12} x_{12}\left[a_{22}\left(\beta_{1} x_{23}-\beta_{2} x_{22}-\beta_{3} x_{21}^{2} x_{22}\right)+a_{32}\left(\gamma_{1} x_{33}-\gamma_{2} x_{32}-\gamma_{3} x_{31}^{2} x_{32}\right)\right] \\
& -a_{42} \eta_{1} x_{43}+a_{42} \eta_{2} x_{42}+a_{42} \eta_{3} x_{41}^{2} x_{42}, \\
\widetilde{\Theta}= & a_{13}\left(\alpha_{4} x_{12}-\alpha_{4} x_{13}-\alpha_{5} x_{14}\right)\left(a_{23} x_{23}+a_{33} x_{33}\right)+a_{13} x_{13}\left[a_{23}\left(\beta_{4} x_{22}-\beta_{4} x_{23}-\beta_{5} x_{24}\right)\right. \\
& \left.+a_{33}\left(\gamma_{4} x_{32}-\gamma_{4} x_{33}-\gamma_{5} x_{34}\right)\right]-a_{43} \eta_{4} x_{42}+a_{43} \eta_{4} x_{43}+a_{43} \eta_{5} x_{44} .
\end{aligned}
$$

Proof For simplicity, we rewrite system (16) as follows:

$$
\left\{\begin{array}{l}
\dot{e}_{1}=\Xi_{1}-a_{41} x_{42}-a_{41} u_{1}, \\
\dot{e}_{2}=\Xi_{2}-a_{42} \eta_{1} x_{43}+a_{42} \eta_{2} x_{42}+a_{42} \eta_{3} x_{41}^{2} x_{42}-a_{42} u_{2}, \\
\dot{e}_{3}=\Xi_{3}-a_{43} \eta_{4} x_{42}+a_{43} \eta_{4} x_{43}+a_{43} \eta_{5} x_{44}-a_{43} u_{3}, \\
\dot{e}_{4}=\Xi_{4}-a_{44} \eta_{6} x_{43}-a_{44} u_{4},
\end{array}\right.
$$

where

$$
\begin{aligned}
\Xi_{1}= & a_{11} x_{12}\left(a_{21} x_{21}+a_{31} x_{31}\right)+a_{11} x_{11}\left(a_{21} x_{22}+a_{31} x_{32}\right), \\
\Xi_{2}= & a_{12}\left(\alpha_{1} x_{13}-\alpha_{2} x_{12}-\alpha_{3} x_{11}^{2} x_{12}\right)\left(a_{22} x_{22}+a_{32} x_{32}\right) \\
& +a_{12} x_{12}\left[a_{22}\left(\beta_{1} x_{23}-\beta_{2} x_{22}-\beta_{3} x_{21}^{2} x_{22}\right)+a_{32}\left(\gamma_{1} x_{33}-\gamma_{2} x_{32}-\gamma_{3} x_{31}^{2} x_{32}\right)\right], \\
\Xi_{3}= & a_{13}\left(\alpha_{4} x_{12}-\alpha_{4} x_{13}-\alpha_{5} x_{14}\right)\left(a_{23} x_{23}+a_{33} x_{33}\right) \\
& +a_{13} x_{13}\left[a_{23}\left(\beta_{4} x_{22}-\beta_{4} x_{23}-\beta_{5} x_{24}\right)+a_{33}\left(\gamma_{4} x_{32}-\gamma_{4} x_{33}-\gamma_{5} x_{34}\right)\right], \\
\Xi_{4}= & a_{14} \alpha_{6} x_{13}\left(a_{24} x_{24}+a_{34} x_{34}\right)+a_{14} x_{14}\left(a_{24} \beta_{6} x_{23}+a_{34} \gamma_{6} x_{33}\right) .
\end{aligned}
$$

Consider the following Lyapunov function:

$$
V(e(t))=V\left(e_{1}, e_{2}, e_{3}, e_{4}\right)=\frac{1}{2}\left(e_{1}^{2}+e_{2}^{2}+e_{3}^{2}+e_{4}^{2}\right) .
$$

Calculating the upper right Dini derivative of $V$ along the trajectory of (18), we have

$$
\begin{aligned}
D^{+} V= & e_{1} \dot{e}_{1}+e_{2} \dot{e}_{2}+e_{3} \dot{e}_{3}+e_{4} \dot{e}_{4} \\
= & e_{1}\left(\Xi_{1}-a_{41} x_{42}-a_{41} u_{1}\right)+e_{2}\left(\Xi_{2}-a_{42} \eta_{1} x_{43}+a_{42} \eta_{2} x_{42}+a_{42} \eta_{3} x_{41}^{2} x_{42}-a_{42} u_{2}\right) \\
& +e_{3}\left(\Xi_{3}-a_{43} \eta_{4} x_{42}+a_{43} \eta_{4} x_{43}+a_{43} \eta_{5} x_{44}-a_{43} u_{3}\right) \\
& +e_{4}\left(\Xi_{4}-a_{44} \eta_{6} x_{43}-a_{44} u_{4}\right) .
\end{aligned}
$$

On the basis of (17), by direct computing, we have

$$
\begin{aligned}
& \Xi_{1}-a_{41} x_{42}-a_{41} u_{1}=-e_{1}-\alpha_{1} e_{2}+\eta_{1} e_{4}, \\
& \Xi_{2}-a_{42} \eta_{1} x_{43}+a_{42} \eta_{2} x_{42}+a_{42} \eta_{3} x_{41}^{2} x_{42}-a_{42} u_{2}=-e_{2}-\alpha_{2} e_{3}+\alpha_{1} e_{1}, \\
& \Xi_{3}-a_{43} \eta_{4} x_{42}+a_{43} \eta_{4} x_{43}+a_{43} \eta_{5} x_{44}-a_{43} u_{3}=-e_{3}-\alpha_{3} e_{4}+\alpha_{2} e_{2}, \\
& \Xi_{4}-a_{44} \eta_{6} x_{43}-a_{44} u_{4}=-e_{4}-\eta_{1} e_{1}+\alpha_{3} e_{3} .
\end{aligned}
$$


Together with (20) and (21),

$$
\begin{aligned}
D^{+} V= & e_{1}\left(-e_{1}-\alpha_{1} e_{2}+\eta_{1} e_{4}\right)+e_{2}\left(-e_{2}-\alpha_{2} e_{3}+\alpha_{1} e_{1}\right)+e_{3}\left(-e_{3}-\alpha_{3} e_{4}+\alpha_{2} e_{2}\right) \\
& +e_{4}\left(-e_{4}-\eta_{1} e_{1}+\alpha_{3} e_{3}\right) \\
= & -e_{1}^{2}-e_{2}^{2}-e_{3}^{2}-e_{4}^{2} \\
= & -e^{T} e
\end{aligned}
$$

where $e=\left(e_{1}, e_{2}, e_{3}, e_{4}\right)^{T}$.

Let $t>0$ be arbitrarily given; integrating the above equation (22) from 0 to $t$, we have

$$
\int_{0}^{t}\|e(s)\|^{2} \mathrm{~d} s=\int_{0}^{t}-\dot{V} \mathrm{~d} s=V(e(0))-V(e(t)) \leq V(e(0))
$$

where $\|\cdot\|$ is the Euclidean vector norm.

Applying Barbalat's lemma, we have $\|e(t)\|^{2} \rightarrow 0$ as $t \rightarrow+\infty$. Hence, $\left(e_{1}, e_{2}, e_{3}, e_{4}\right) \rightarrow$ $(0,0,0,0)$ as $t \rightarrow+\infty$. It implies that the drive systems (10)-(12) compound synchronize with the response system (13).

Remark 6 In Theorem 1, since we have the unique structural design in compound synchronization, hence the control input used in Theorem 1 is a little more complicated. Just as in [8], the nonlinearity of the designed control law in the compound synchronization scheme is high. How to design some less conservative criteria for compound synchronization scheme? This issue will be the topic of future research.

Next, some corollaries follow easily from Theorem 1 . These corollaries provide simpler criteria for selecting the applicable control laws with easy implementation.

Corollary 1 The drive systems (10) and (11) function projective synchronize with the response system (13) if the control input is designed as

$$
\left\{\begin{aligned}
u_{1}= & \frac{1}{a_{41}}\left[a_{11} x_{11}\left(a_{21} x_{22}+a_{21} x_{21}\right)+a_{11} a_{21} x_{12} x_{21}+\alpha_{1} a_{12} a_{22} x_{12} x_{22}\right. \\
& \left.-\eta_{1} a_{14} a_{24} x_{14} x_{24}-\left(a_{41}+\alpha_{1} a_{42}\right) x_{42}+\eta_{1} a_{44} x_{44}\right], \\
u_{2}= & \frac{1}{a_{42}}\left(\Theta-\alpha_{1}\left[a_{11} a_{21} x_{11} x_{21}-a_{41} x_{41}\right]+\left[a_{12} a_{22} x_{12} x_{22}-a_{42} x_{42}\right]-\alpha_{2} a_{43} x_{43}\right), \\
u_{3}= & \frac{1}{a_{43}}\left(\widetilde{\Theta}-\alpha_{2}\left[a_{12} a_{22} x_{12} x_{22}-a_{42} x_{42}\right]+\left[a_{13} a_{23} x_{13} x_{23}-a_{43} x_{43}\right]\right. \\
& \left.+\alpha_{3}\left[a_{14} a_{24} x_{14} x_{24}-a_{44} x_{44}\right]\right), \\
u_{4}= & \frac{1}{a_{44}}\left[\eta_{1} a_{11} a_{21} x_{11} x_{21}-\alpha_{3} a_{13} a_{23} x_{13} x_{23}+\alpha_{6} a_{14} a_{24} x_{13} x_{24}\right. \\
& \left.+a_{14} x_{14}\left(\beta_{6} a_{24} x_{23}+a_{24} x_{24}\right)-\eta_{1} a_{41} x_{41}-\alpha_{4} a_{43} x_{43}-\eta_{6} a_{44} x_{43}-a_{44} x_{44}\right],
\end{aligned}\right.
$$

where

$$
\begin{aligned}
\Theta= & a_{12} a_{22} x_{22}\left(\alpha_{1} x_{13}-\alpha_{2} x_{12}-\alpha_{3} x_{11}^{2} x_{12}\right)+a_{12} a_{22} x_{12}\left(\beta_{1} x_{23}-\beta_{2} x_{22}-\beta_{3} x_{21}^{2} x_{22}\right) \\
& -a_{42} \eta_{1} x_{43}+a_{42} \eta_{2} x_{42}+a_{42} \eta_{3} x_{41}^{2} x_{42}, \\
\widetilde{\Theta}= & a_{13} a_{23} x_{23}\left(\alpha_{4} x_{12}-\alpha_{4} x_{13}-\alpha_{5} x_{14}\right)+a_{13} a_{23} x_{13}\left(\beta_{4} x_{22}-\beta_{4} x_{23}-\beta_{5} x_{24}\right) \\
& -a_{43} \eta_{4} x_{42}+a_{43} \eta_{4} x_{43}+a_{43} \eta_{5} x_{44} .
\end{aligned}
$$


Corollary 2 The drive systems (10) and (12) function projective synchronize with the response system (13) if the control input is designed as

$$
\left\{\begin{aligned}
u_{1}= & \frac{1}{a_{41}}\left[a_{11} x_{11}\left(a_{31} x_{32}+a_{31} x_{31}\right)+a_{11} a_{31} x_{12} x_{31}+\alpha_{1} a_{12} a_{32} x_{12} x_{32}\right. \\
& \left.-\eta_{1} a_{14} a_{34} x_{14} x_{34}-\left(a_{41}+\alpha_{1} a_{42}\right) x_{42}+\eta_{1} a_{44} x_{44}\right], \\
u_{2}= & \frac{1}{a_{42}}\left(\Theta-\alpha_{1}\left[a_{11} a_{31} x_{11} x_{31}-a_{41} x_{41}\right]+\left[a_{12} a_{32} x_{12} x_{32}-a_{42} x_{42}\right]\right. \\
& \left.+\alpha_{2}\left[a_{13} a_{33} x_{13} x_{33}-a_{43} x_{43}\right]\right), \\
u_{3}= & \frac{1}{a_{43}}\left(\widetilde{\Theta}-\alpha_{2}\left[a_{12} a_{32} x_{12} x_{32}-a_{42} x_{42}\right]+\left[a_{13} a_{33} x_{13} x_{33}-a_{43} x_{43}\right]\right. \\
& \left.+\alpha_{3}\left[a_{14} a_{34} x_{14} x_{34}-a_{44} x_{44}\right]\right), \\
u_{4}= & \frac{1}{a_{44}}\left[\eta_{1} a_{11} a_{31} x_{11} x_{31}-\alpha_{3} a_{13} a_{33} x_{13} x_{33}+\alpha_{6} a_{14} a_{34} x_{13} x_{34}\right. \\
& \left.+a_{14} x_{14}\left(\gamma_{6} a_{34} x_{33}+a_{34} x_{34}\right)-\eta_{1} a_{41} x_{41}-\alpha_{4} a_{43} x_{43}-\eta_{6} a_{44} x_{43}-a_{44} x_{44}\right]
\end{aligned}\right.
$$

where

$$
\begin{aligned}
\Theta= & a_{12} a_{32} x_{32}\left(\alpha_{1} x_{13}-\alpha_{2} x_{12}-\alpha_{3} x_{11}^{2} x_{12}\right)+a_{12} a_{32} x_{12}\left(\gamma_{1} x_{33}-\gamma_{2} x_{32}-\gamma_{3} x_{31}^{2} x_{32}\right) \\
& -a_{42} \eta_{1} x_{43}+a_{42} \eta_{2} x_{42}+a_{42} \eta_{3} x_{41}^{2} x_{42}, \\
\widetilde{\Theta}= & a_{13} a_{33} x_{33}\left(\alpha_{4} x_{12}-\alpha_{4} x_{13}-\alpha_{5} x_{14}\right)+a_{13} a_{33} x_{13}\left(\gamma_{4} x_{32}-\gamma_{4} x_{33}-\gamma_{5} x_{34}\right) \\
& -a_{43} \eta_{4} x_{42}+a_{43} \eta_{4} x_{43}+a_{43} \eta_{5} x_{44} .
\end{aligned}
$$

Corollary 3 System (13) is asymptotically stabilizable if the control input is designed as

$$
\left\{\begin{array}{l}
u_{1}=\frac{1}{a_{41}}\left[-\left(a_{41}+\alpha_{1} a_{42}\right) x_{42}+\eta_{1} a_{44} x_{44}\right], \\
u_{2}=\frac{1}{a_{42}}\left[-a_{42} \eta_{1} x_{43}+a_{42} \eta_{2} x_{42}+a_{42} \eta_{3} x_{41}^{2} x_{42}-a_{41} x_{41}-a_{42} x_{42}-a_{43} x_{43}\right], \\
u_{3}=\frac{1}{a_{43}}\left[-a_{43} \eta_{4} x_{42}+a_{43} \eta_{4} x_{43}+a_{43} \eta_{5} x_{44}-a_{42} x_{42}-a_{43} x_{43}-a_{44} x_{44}\right], \\
u_{4}=\frac{1}{a_{44}}\left[-\eta_{1} a_{41} x_{41}-\alpha_{4} a_{43} x_{43}-\eta_{6} a_{44} x_{43}-a_{44} x_{44}\right] .
\end{array}\right.
$$

Remark 7 It is well known that one common problem on the compound of multiple drive systems is that the compound signal often is asymptotically stable or emanative. As a result, the dynamic behaviors would definitely do harm for the engineering designers, since dynamic evolution evoked by the compound of multiple drive systems is either too easy or completely useless. However, it is worth noting that the compound of multiple drive systems in this paper can be still chaotic; accordingly, the dynamic evolution has the ability of being pseudorandom and sensitive to the initial value, and it has unpredictability of path. This can be well applied to secure communication.

Remark 8 As is well known, the memristive oscillator system is still quite incipient and to the best of our knowledge there is not a lot of results for exploring the synchronization control. The proposed control scheme in this paper can offer some valuable guidance to the research and application of memristor devices.

Now, we give a numerical example to illustrate the superiority of theoretical results via computer simulations. Assume $\alpha_{1}=\beta_{1}=\gamma_{1}=\eta_{1}=16.4, \alpha_{2}=\beta_{2}=\gamma_{2}=\eta_{2}=3.28, \alpha_{3}=\beta_{3}=$ $\gamma_{3}=\eta_{3}=19.7, \alpha_{4}=\beta_{4}=\gamma_{4}=\eta_{4}=1, \alpha_{5}=\beta_{5}=\gamma_{5}=\eta_{5}=1, \alpha_{6}=\beta_{6}=\gamma_{6}=\eta_{6}=15$. Choose $A_{1}=\operatorname{diag}\left(a_{11}, a_{12}, a_{13}, a_{14}\right)=\operatorname{diag}(1,1,1,1), A_{2}=\operatorname{diag}\left(a_{21}, a_{22}, a_{23}, a_{24}\right)=\operatorname{diag}(1,1,1,1), A_{3}=$ $\operatorname{diag}\left(a_{31}, a_{32}, a_{33}, a_{34}\right)=\operatorname{diag}(1,1,1,1), A_{4}=\operatorname{diag}\left(a_{41}, a_{42}, a_{43}, a_{44}\right)=\operatorname{diag}(1,1,1,1)$, then the 
control input in the scheme of compound synchronization can be designed as

$$
\left\{\begin{aligned}
u_{1}= & x_{11}\left(x_{22}+x_{32}+x_{21}+x_{31}\right)+x_{12}\left(x_{21}+x_{31}+16.4 x_{22}+16.4 x_{32}\right) \\
& -16.4 x_{14}\left(x_{24}+x_{34}\right)-17.4 x_{42}+16.4 x_{44}, \\
u_{2}= & \Theta-16.4\left[x_{11}\left(x_{21}+x_{31}\right)-x_{41}\right]+\left[x_{12}\left(x_{22}+x_{32}\right)-x_{42}\right] \\
& +3.28\left[x_{13}\left(x_{23}+x_{33}\right)-x_{43}\right], \\
u_{3}= & \widetilde{\Theta}-3.28\left[x_{12}\left(x_{22}+x_{32}\right)-x_{42}\right]+\left[x_{13}\left(x_{23}+x_{33}\right)-x_{43}\right] \\
& +19.7\left[x_{14}\left(x_{24}+x_{34}\right)-x_{44}\right], \\
u_{4}= & 16.4 x_{11}\left(x_{21}+x_{31}\right)-19.7 x_{13}\left(x_{23}+x_{33}\right)+15 x_{13}\left(x_{24}+x_{34}\right) \\
& +x_{14}\left(15 x_{23}+x_{24}\right)+x_{14}\left(15 x_{33}+x_{34}\right)-16.4 x_{41}-16 x_{43}-x_{44},
\end{aligned}\right.
$$

where

$$
\begin{aligned}
\Theta= & \left(16.4 x_{13}-3.28 x_{12}-19.7 x_{11}^{2} x_{12}\right)\left(x_{22}+x_{32}\right)+x_{12}\left[\left(16.4 x_{23}-3.28 x_{22}-19.7 x_{21}^{2} x_{22}\right)\right. \\
& \left.+\left(16.4 x_{33}-3.28 x_{32}-19.7 x_{31}^{2} x_{32}\right)\right]-16.4 x_{43}+3.28 x_{42}+19.7 x_{41}^{2} x_{42}, \\
\widetilde{\Theta}= & \left(x_{12}-x_{13}-x_{14}\right)\left(x_{23}+x_{33}\right)+x_{13}\left[\left(x_{22}-x_{23}-x_{24}\right)+\left(x_{32}-x_{33}-x_{34}\right)\right] \\
& -x_{42}+x_{43}+x_{44} .
\end{aligned}
$$

Remark 9 Here we express an added illustration on the parameters selection $\alpha_{i}, \beta_{i}, \gamma_{i}$, $\eta_{i}(i=1,2, \ldots, 6)$ in the numerical example above. These values used as parameters have many benefits. One of the most evident features is that the nonlinear dynamics of memristor oscillator (10) are very rich.

Simulation result of the compound of three drive systems (10)-(12) is depicted in Figure 4. The computer simulation suggests the compound of three drive systems (10)-(12) has a strange attractor, as shown in Figure 4, which has verified that the compound of drive systems (10)-(12) remains chaotic. Meanwhile, according to Theorem 1, the compound of three drive systems (10)-(12) can achieve synchronization of the response system (13). Figure 5 depicts the time response of the synchronization error $e(t)=\left(e_{1}(t), e_{2}(t), e_{3}(t), e_{4}(t)\right)^{T}$.

\section{The application in secure communication}

As an application of the results obtained in the preceding section, secure communication via compound synchronization is discussed in this section.

Let $\zeta(t)=r_{1}(t)\left[r_{2}(t)+r_{3}(t)\right]$ be the message signal to be received. Corresponding to this message signal, adding it to the right of the first equation for the transmitter (three drive systems), we have

$$
\begin{aligned}
& \left\{\begin{array}{l}
\dot{x}_{11}=x_{12}+r_{1}, \\
\dot{x}_{12}=\alpha_{1} x_{13}-\alpha_{2} x_{12}-\alpha_{3} x_{11}^{2} x_{12}, \\
\dot{x}_{13}=\alpha_{4} x_{12}-\alpha_{4} x_{13}-\alpha_{5} x_{14}, \\
\dot{x}_{14}=\alpha_{6} x_{13}
\end{array}\right. \\
& \left\{\begin{array}{l}
\dot{x}_{21}=x_{22}+r_{2} \\
\dot{x}_{22}=\beta_{1} x_{23}-\beta_{2} x_{22}-\beta_{3} x_{21}^{2} x_{22}, \\
\dot{x}_{23}=\beta_{4} x_{22}-\beta_{4} x_{23}-\beta_{5} x_{24}, \\
\dot{x}_{24}=\beta_{6} x_{23}
\end{array}\right.
\end{aligned}
$$




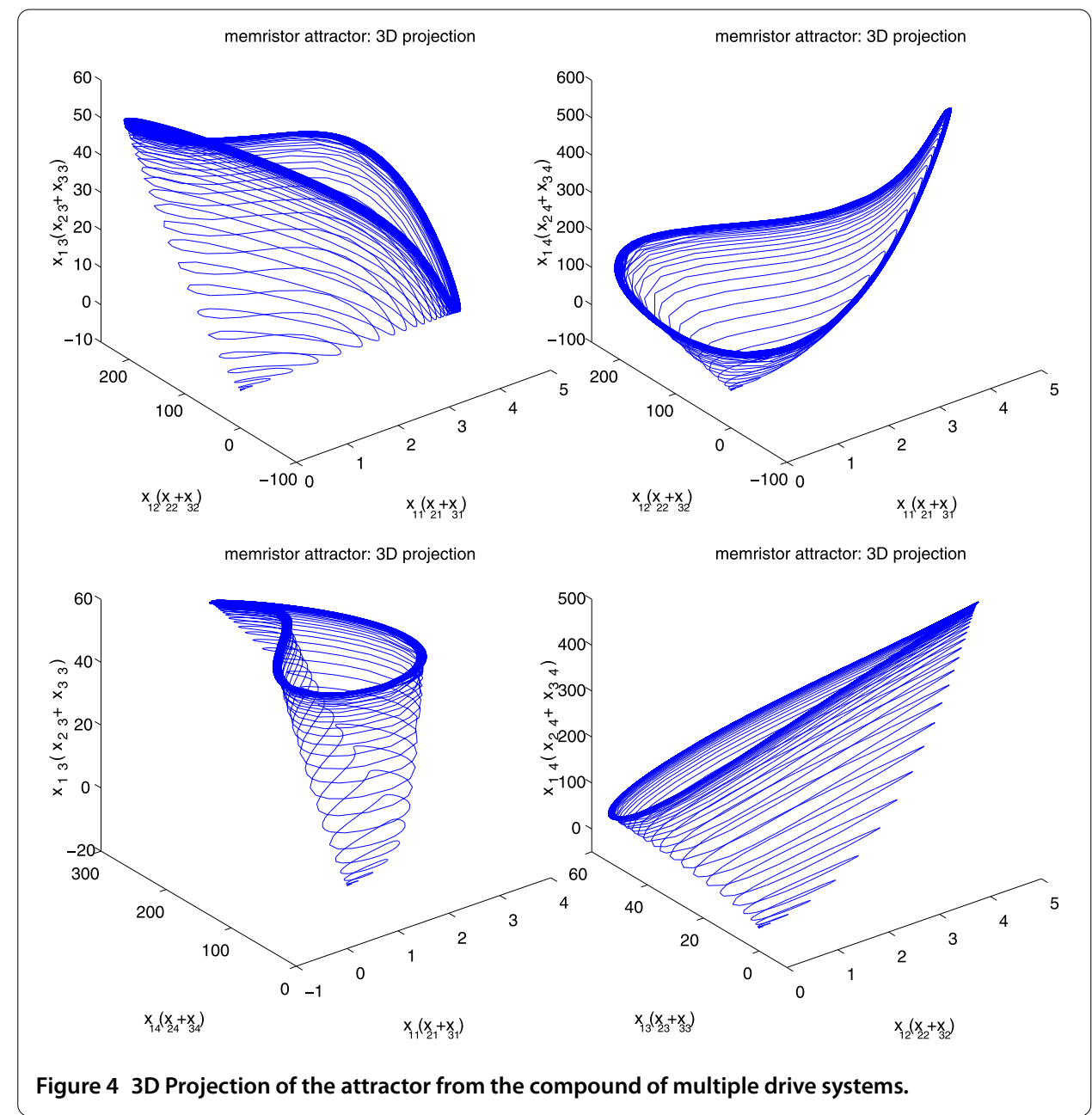

$$
\left\{\begin{array}{l}
\dot{x}_{31}=x_{32}+r_{3} \\
\dot{x}_{32}=\gamma_{1} x_{33}-\gamma_{2} x_{32}-\gamma_{3} x_{31}^{2} x_{32} \\
\dot{x}_{33}=\gamma_{4} x_{32}-\gamma_{4} x_{33}-\gamma_{5} x_{34} \\
\dot{x}_{34}=\gamma_{6} x_{33}
\end{array}\right.
$$

Select the output $x_{11}$ of system (23), the output $x_{21}$ of system (24), the output $x_{31}$ of system (25) as the transmitted signals. In our designed scheme, denote $A_{1}=\operatorname{diag}\left(a_{11}, a_{12}, a_{13}\right.$, $\left.a_{14}\right), A_{2}=\operatorname{diag}\left(a_{21}, a_{22}, a_{23}, a_{24}\right), A_{3}=\operatorname{diag}\left(a_{31}, a_{32}, a_{33}, a_{34}\right), A_{4}=\operatorname{diag}\left(a_{41}, a_{42}, a_{43}, a_{44}\right)$, then construct the receiver as follows:

$$
\left\{\begin{array}{l}
\dot{x}_{41}=x_{42}+r_{4}+u_{1}, \\
\dot{x}_{42}=\eta_{1} x_{43}-\eta_{2} x_{42}-\eta_{3} x_{41}^{2} x_{42}+u_{2}, \\
\dot{x}_{43}=\eta_{4} x_{42}-\eta_{4} x_{43}-\eta_{5} x_{44}+u_{3}, \\
\dot{x}_{44}=\eta_{6} x_{43}+u_{4}, \\
\dot{r}_{4}=k\left[a_{11} x_{11}\left(a_{21} x_{21}+a_{31} x_{31}\right)-a_{41} x_{41}\right],
\end{array}\right.
$$

where $r_{4}$ is the message signal to be recovered, $k$ is a parameter. 

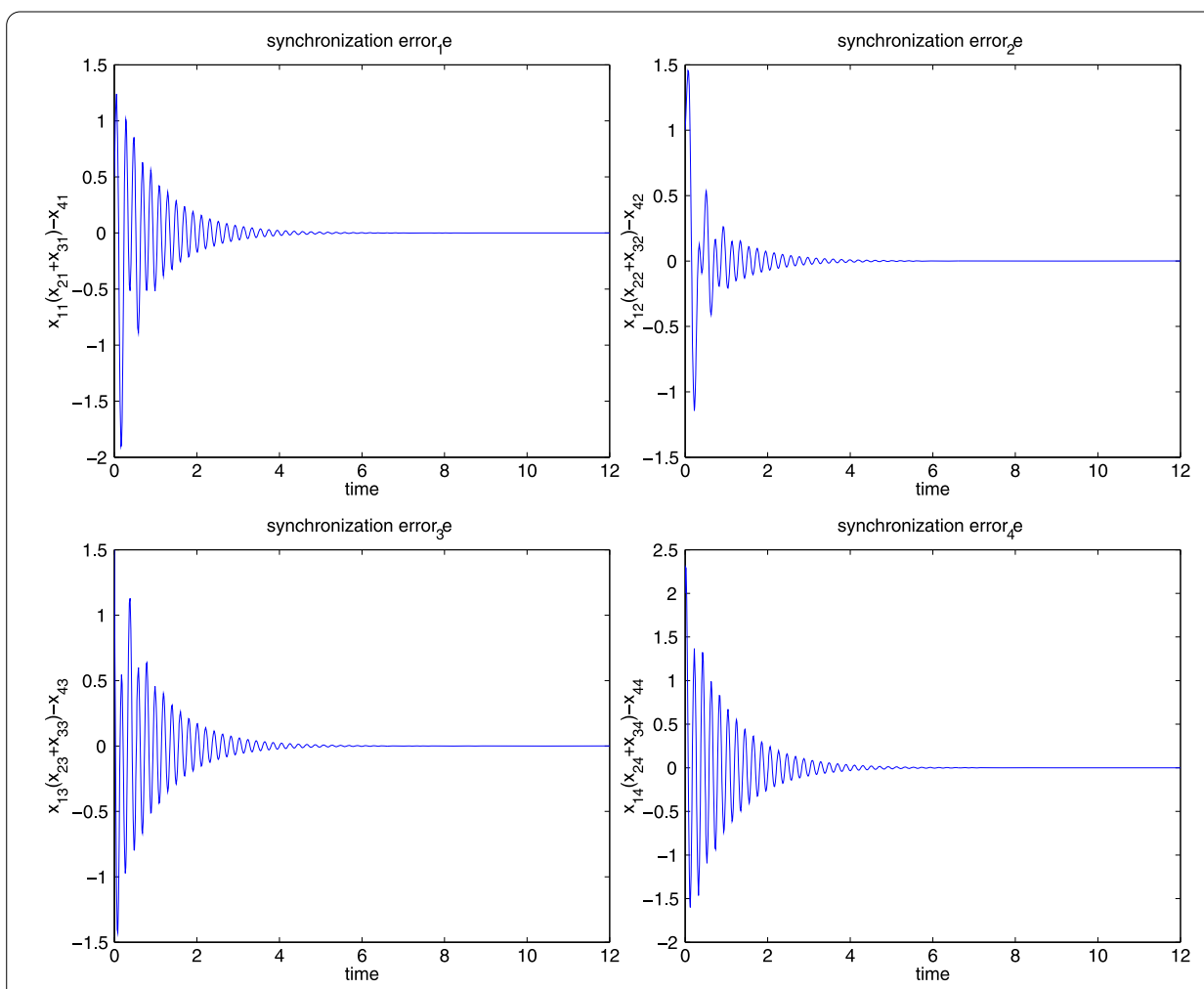

Figure 5 Time response curve for synchronization error $e(t)=\left(e_{1}(t), e_{2}(t), e_{3}(t), e_{4}(t)\right)^{T}$.

Let the tracking error be

$$
\left\{\begin{array}{l}
e_{1}=a_{11} x_{11}\left(a_{21} x_{21}+a_{31} x_{31}\right)-a_{41} x_{41}, \\
e_{2}=a_{12} x_{12}\left(a_{22} x_{22}+a_{32} x_{32}\right)-a_{42} x_{42}, \\
e_{3}=a_{13} x_{13}\left(a_{23} x_{23}+a_{33} x_{33}\right)-a_{43} x_{43}, \\
e_{4}=a_{14} x_{14}\left(a_{24} x_{24}+a_{34} x_{34}\right)-a_{44} x_{44}, \\
e_{5}=r_{1}\left(r_{2}+r_{3}\right)-r_{4},
\end{array}\right.
$$

then the error dynamics is

$$
\left\{\begin{array}{l}
\dot{e}_{1}=a_{11} \dot{x}_{11}\left(a_{21} x_{21}+a_{31} x_{31}\right)+a_{11} x_{11}\left(a_{21} \dot{x}_{21}+a_{31} \dot{x}_{31}\right)-a_{41} \dot{x}_{41}, \\
\dot{e}_{2}=a_{12} \dot{x}_{12}\left(a_{22} x_{22}+a_{32} x_{32}\right)+a_{12} x_{12}\left(a_{22} \dot{x}_{22}+a_{32} \dot{x}_{32}\right)-a_{42} \dot{x}_{42}, \\
\dot{e}_{3}=a_{13} \dot{x}_{13}\left(a_{23} x_{23}+a_{33} x_{33}\right)+a_{13} x_{13}\left(a_{23} \dot{x}_{23}+a_{33} \dot{x}_{33}\right)-a_{43} \dot{x}_{43}, \\
\dot{e}_{4}=a_{14} \dot{x}_{14}\left(a_{24} x_{24}+a_{34} x_{34}\right)+a_{14} x_{14}\left(a_{24} \dot{x}_{24}+a_{34} \dot{x}_{34}\right)-a_{44} \dot{x}_{44} \\
\dot{e}_{5}=\frac{\mathrm{d} r_{1}\left(r_{2}+r_{3}\right)}{\mathrm{d} t}-k e_{1} .
\end{array}\right.
$$

Since the eigenfrequency of the message signal $\zeta(t)=r_{1}(t)\left[r_{2}(t)+r_{3}(t)\right]$ is much smaller than the oscillating frequency of the chaotic system in practice, then $\frac{\mathrm{d} r_{1}\left(r_{2}+r_{3}\right)}{\mathrm{d} t}-k e_{1} \approx-k e_{1}$.

Theorem 2 If control input is designed as Theorem 1, and the message update law of $r_{4}$ satisfies

$$
\frac{\mathrm{d} r_{4}}{\mathrm{~d} t}=\frac{1}{a_{41}-k}\left[a_{11} \frac{\mathrm{d} r_{1}\left(a_{21} x_{21}+a_{31} x_{31}\right)}{\mathrm{d} t}+a_{11} \frac{\mathrm{d} x_{11}\left(a_{21} r_{2}+a_{31} r_{3}\right)}{\mathrm{d} t}-k \frac{\mathrm{d} r_{1}\left(r_{2}+r_{3}\right)}{\mathrm{d} t}\right],
$$


then $\left(e_{1}, e_{2}, e_{3}, e_{4}, e_{5}\right) \rightarrow(0,0,0,0,0)$ as $t \rightarrow+\infty$. It implies that $r_{4}$ can recover the message signal $\zeta(t)=r_{1}(t)\left[r_{2}(t)+r_{3}(t)\right]$.

Proof Let

$$
V(e(t))=V\left(e_{1}, e_{2}, e_{3}, e_{4}, e_{5}\right)=\frac{1}{2}\left(e_{1}^{2}+e_{2}^{2}+e_{3}^{2}+e_{4}^{2}+e_{5}^{2}\right),
$$

then the proof of Theorem 2 is very similar to the proof of Theorem 1 and thus is omitted here for brevity.

Remark 10 In the preceding discussion, we only touch upon the secure communication via compound synchronization from a theoretical analysis point. As for how to encode the message for secure communication via compound synchronization, one might conduct further research. Of course, this is another issue, which is on the level of engineering practice.

Remark 11 In the existing literature, the secure communication via compound synchronization has rarely been studied. By the above discussions in this paper, it is easy to see that in theory, compound synchronization can greatly improve the complexity level of the driving signal and the modulation scheme used. In [8], secure communication via the compound design of the memristor chaotic oscillator system has been discussed. It is worth noting that the result of Theorem 2 in [8] leaves room for improvement. In [8], the message update law of the message signal to be recovered is described as the first and second derivatives of the states and the message signals to be received. The message update law in our criterion contains just the first derivative of the states and the message signals to be received. And consequently, the condition, which depends only on the first derivative of the states and the message signals to be received, is easy to check. Therefore, our method may be good in theory. In addition, the function $V(e(t))$ in Theorem 2 of Sun et al. [8] is $V(e(t))=V\left(e_{1}, e_{2}, e_{3}, e_{4}, e_{5}\right)=\frac{1}{2}\left(e_{1}^{2}+e_{2}^{2}+e_{3}^{2}\right)+\frac{1}{3}\left(e_{4}+e_{5}\right)^{2}$. Obviously, strictly speaking, this function $V(e(t))=V\left(e_{1}, e_{2}, e_{3}, e_{4}, e_{5}\right)=\frac{1}{2}\left(e_{1}^{2}+e_{2}^{2}+e_{3}^{2}\right)+\frac{1}{3}\left(e_{4}+e_{5}\right)^{2}$ is a nonnegative function, not a Lyapunov function, since it fails to meet positive definiteness. Thus, the derived corresponding results based on the Lyapunov theory in [8] are considered conservative.

\section{Concluding remarks}

In this paper, we have applied the compound of multiple drive systems to investigate the synchronization control of a class of fourth-order memristor oscillator. The proposed control scheme theoretically guarantees the good control performance. The main disadvantage of the obtained results lies in the highly nonlinear nature of the designed controller. Finally, we have suggested an approach for a potential application of memristor oscillators in secure communication. Therefore, the derived results may offer useful and broad-range applications in electronics. 


\section{Author details}

${ }^{1}$ College of Mathematics and Statistics, Hubei Normal University, Huangshi, 435002, China. ${ }^{2}$ Institute for Information and System Science, Xi'an Jiaotong University, Xi'an, 710049, China. ${ }^{3}$ School of Automation, Huazhong University of Science and Technology, Wuhan, 430074, China.

\section{Acknowledgements}

The authors appreciate the constructive comments from the Editor and anonymous referees with which the paper has been improved. The work is supported by the Natural Science Foundation of China under Grant 61304057 and the Project funded by China Postdoctoral Science Foundation, the Postdoctoral Research Program of Shaanxi Province of China.

\section{Received: 25 December 2013 Accepted: 26 March 2014 Published: 03 Apr 2014}

\section{References}

1. Bao, BC, Liu, Z, Xu, JP: Steady periodic memristor oscillator with transient chaotic behaviours. Electron. Lett. 46(3), 237-238 (2010)

2. Bao, BC, Liu, Z, Xu, JP: Transient chaos in smooth memristor oscillator. Chin. Phys. B 19(3), 030510 (2010)

3. Corinto, F, Ascoli, A, Gilli, M: Nonlinear dynamics of memristor oscillators. IEEE Trans. Circuits Syst. I, Regul. Pap. 58(6), 1323-1336 (2011)

4. Itoh, M, Chua, LO: Memristor oscillators. Int. J. Bifurc. Chaos 18(11), 3183-3206 (2008)

5. Li, ZJ, Zeng, YC: A memristor oscillator based on a twin-T network. Chin. Phys. B 22(4), 040502 (2013)

6. Muthuswamy, B, Kokate, PP: Memristor based chaotic circuits. IETE Tech. Rev. 26(6), 417-429 (2009)

7. Riaza, R: First order mem-circuits: modeling, nonlinear oscillations and bifurcation. IEEE Trans. Circuits Syst. I, Regul. Pap. 60(6), 1570-1583 (2013)

8. Sun, JW, Shen, Y, Yin, Q, Xu, CJ: Compound synchronization of four memristor chaotic oscillator systems and secure communication. Chaos 23(1), 013140 (2013)

9. Talukdar, A, Radwan, AG, Salama, KN: Generalized model for memristor-based Wien family oscillators. Microelectron. J. 42(9), 1032-1038 (2011)

10. Talukdar, A, Radwan, AG, Salama, KN: Non linear dynamics of memristor based 3rd order oscillatory system. Microelectron. J. 43(3), 169-175 (2012)

11. Wu, AL, Wen, SP, Zeng, ZG: Synchronization control of a class of memristor-based recurrent neural networks. Inf. Sci. 183(1), 106-116 (2012)

12. $\mathrm{Wu}, \mathrm{AL}$, Zeng, ZG: Dynamic behaviors of memristor-based recurrent neural networks with time-varying delays. Neural Netw. 36, 1-10 (2012)

13. $\mathrm{Wu}, \mathrm{AL}$, Zeng, ZG: Exponential stabilization of memristive neural networks with time delays. IEEE Trans. Neural Netw. Learn. Syst. 23(12), 1919-1929 (2012)

14. Choi, YP, Ha, SY, Yun, SB: Complete synchronization of Kuramoto oscillators with finite inertia. Physica D 240(1), 32-44 (2011)

15. Li, FF, Lu, XW: Complete synchronization of temporal Boolean networks. Neural Netw. 44, 72-77 (2013)

16. Ma, J, Li, F, Huang, L, Jin, WY: Complete synchronization, phase synchronization and parameters estimation in a realistic chaotic system. Commun. Nonlinear Sci. Numer. Simul. 16(9), 3770-3785 (2011)

17. $\mathrm{Wu}, \mathrm{XJ}, \mathrm{Lu}, \mathrm{HT}$ : Generalized function projective (lag, anticipated and complete) synchronization between two different complex networks with nonidentical nodes. Commun. Nonlinear Sci. Numer. Simul. 17(7), 3005-3021 (2012)

18. Yao, CG, Zhao, Q, Yu, J: Complete synchronization induced by disorder in coupled chaotic lattices. Phys. Lett. A 377(5), 370-377 (2013)

19. Chen, $Q$, Ren, XM, Na, J: Robust anti-synchronization of uncertain chaotic systems based on multiple-kernel least squares support vector machine modeling. Chaos Solitons Fractals 44(12), 1080-1088 (2011)

20. Fu, GY, Li, ZS: Robust adaptive anti-synchronization of two different hyperchaotic systems with external uncertainties Commun. Nonlinear Sci. Numer. Simul. 16(1), 395-401 (2011)

21. Liu, ST, Liu, P: Adaptive anti-synchronization of chaotic complex nonlinear systems with unknown parameters. Nonlinear Anal., Real World Appl. 12(6), 3046-3055 (2011)

22. $\mathrm{Wu}, \mathrm{YQ}, \mathrm{Li}, \mathrm{CP}$, Yang, AL, Song, LJ, Wu, YJ: Pinning adaptive anti-synchronization between two general complex dynamical networks with non-delayed and delayed coupling. Appl. Math. Comput. 218(14), 7445-7452 (2012)

23. Zhang, GD, Shen, Y, Wang, LM: Global anti-synchronization of a class of chaotic memristive neural networks with time-varying delays. Neural Netw. 46, 1-8 (2013)

24. Zhao, HY, Zhang, Q: Global impulsive exponential anti-synchronization of delayed chaotic neural networks. Neurocomputing 74(4), 563-567 (2011)

25. Li, D, Li, XL, Cui, D, Li, ZH: Phase synchronization with harmonic wavelet transform with application to neuronal populations. Neurocomputing 74(17), 3389-3403 (2011)

26. Odibat, Z: A note on phase synchronization in coupled chaotic fractional order systems. Nonlinear Anal., Real World Appl. 13(2), 779-789 (2012)

27. Taghvafard, H, Erjaee, GH: Phase and anti-phase synchronization of fractional order chaotic systems via active control. Commun. Nonlinear Sci. Numer. Simul. 16(10), 4079-4088 (2011)

28. Feng, JW, Dai, AD, Xu, C, Wang, JY: Designing lag synchronization schemes for unified chaotic systems. Comput. Math. Appl. 61(8), 2123-2128 (2011)

29. Guo, WL: Lag synchronization of complex networks via pinning control. Nonlinear Anal., Real World Appl. 12(5), 2579-2585 (2011)

30. Ji, DH, Jeong, SC, Park, JH, Lee, SM, Won, SC: Adaptive lag synchronization for uncertain complex dynamical network with delayed coupling. Appl. Math. Comput. 218(9), 4872-4880 (2012)

31. Pourdehi, S, Karimaghaee, P, Karimipour, D: Adaptive controller design for lag-synchronization of two non-identical time-delayed chaotic systems with unknown parameters. Phys. Lett. A 375(17), 1769-1778 (2011)

32. Wang, LP, Yuan, ZT, Chen, XH, Zhou, ZF: Lag synchronization of chaotic systems with parameter mismatches. Commun. Nonlinear Sci. Numer. Simul. 16(2), 987-992 (2011) 
33. Wang, ZL, Shi, XR: Lag synchronization of two identical Hindmarsh-Rose neuron systems with mismatched parameters and external disturbance via a single sliding mode controller. Appl. Math. Comput. 218(22), 10914-10921 (2012)

34. Xing, ZW, Peng, JG: Exponential lag synchronization of fuzzy cellular neural networks with time-varying delays. J. Franklin Inst. 349(3), 1074-1086 (2012)

35. Yang, XS, Zhu, QX, Huang, CX: Generalized lag-synchronization of chaotic mix-delayed systems with uncertain parameters and unknown perturbations. Nonlinear Anal., Real World Appl. 12(1), 93-105 (2011)

36. $\mathrm{Yu}, \mathrm{J}, \mathrm{Hu}, \mathrm{C}$, Jiang, HJ, Teng, ZD: Exponential lag synchronization for delayed fuzzy cellular neural networks via periodically intermittent control. Math. Comput. Simul. 82(5), 895-908 (2012)

37. Farivar, F, Shoorehdeli, MA, Nekoui, MA, Teshnehlab, M: Generalized projective synchronization of uncertain chaotic systems with external disturbance. Expert Syst. Appl. 38(5), 4714-4726 (2011)

38. Li, ZB, Zhao, XS: Generalized function projective synchronization of two different hyperchaotic systems with unknown parameters. Nonlinear Anal., Real World Appl. 12(5), 2607-2615 (2011)

39. Si, GQ, Sun, ZY, Zhang, YB, Chen, WQ: Projective synchronization of different fractional-order chaotic systems with non-identical orders. Nonlinear Anal., Real World Appl. 13(4), 1761-1771 (2012)

40. Wang, S, Yu, YG, Wen, GG: Hybrid projective synchronization of time-delayed fractional order chaotic systems. Nonlinear Anal. Hybrid Syst. 11, 129-138 (2014)

41. Wang, XY, Fan, B: Generalized projective synchronization of a class of hyperchaotic systems based on state observer. Commun. Nonlinear Sci. Numer. Simul. 17(2), 953-963 (2012)

42. Wu, XJ, Wang, H, Lu, HT: Hyperchaotic secure communication via generalized function projective synchronization Nonlinear Anal., Real World Appl. 12(2), 1288-1299 (2011)

43. Xiao, JW, Wang, ZW, Miao, WT, Wang, YW: Adaptive pinning control for the projective synchronization of drive-response dynamical networks. Appl. Math. Comput. 219(5), 2780-2788 (2012)

44. Yu, YG, Li, HX: Adaptive hybrid projective synchronization of uncertain chaotic systems based on backstepping design. Nonlinear Anal., Real World Appl. 12(1), 388-393 (2011)

45. Zhou, P, Zhu, W: Function projective synchronization for fractional-order chaotic systems. Nonlinear Anal., Real World Appl. 12(2), 811-816 (2011)

46. Luo, RZ, Wang, YL, Deng, SC: Combination synchronization of three classic chaotic systems using active backstepping design. Chaos 21(4), 043114 (2011)

47. Sun, JW, Shen, Y, Zhang, GD, Xu, CJ, Cui, GZ: Combination-combination synchronization among four identical or different chaotic systems. Nonlinear Dyn. 73(3), 1211-1222 (2013)

48. Grzybowski, JMV, Rafikov, M, Balthazar, JM: Synchronization of the unified chaotic system and application in secure communication. Commun. Nonlinear Sci. Numer. Simul. 14(6), 2793-2806 (2009)

49. Rafikov, M, Balthazar, JM: On control and synchronization in chaotic and hyperchaotic systems via linear feedback control. Commun. Nonlinear Sci. Numer. Simul. 13(7), 1246-1255 (2008)

10.1186/1687-1847-2014-100

Cite this article as: Wu and Zhang: Compound synchronization of fourth-order memristor oscillator. Advances in Difference Equations 2014, 2014:100

\section{Submit your manuscript to a SpringerOpen ${ }^{\circ}$ journal and benefit from:}

- Convenient online submission

Rigorous peer review

- Immediate publication on acceptance

- Open access: articles freely available online

- High visibility within the field

- Retaining the copyright to your article 